Article

\title{
Investigating Coherent Magnetization Control with Ultrashort THz Pulses
}

\author{
Xuan Liu ${ }^{1}{ }^{(D)}$, Emmanuelle Jal ${ }^{1}{ }^{(\mathbb{D})}$, Renaud Delaunay ${ }^{1}$, Romain Jarrier ${ }^{1}$, Gheorghe Sorin Chiuzbaian ${ }^{1}(\mathbb{D}$, \\ Grégory Malinowski ${ }^{2}$ (D), Torsten Golz ${ }^{3}$, Ekaterina Zapolnova ${ }^{3}$, Rui Pan ${ }^{3}$, Nikola Stojanovic ${ }^{3,4}$, Jan Lüning ${ }^{1,+}$ \\ and Boris Vodungbo $1, *$ (D)
}

1 Laboratoire de Chimie Physique-Matière et Rayonnement (LPCMR), Sorbonne Université, Centre National de la Recherche Scientifique (CNRS), 75252 Paris, France; lxsharper@gmail.com (X.L.); emmanuelle.jal@sorbonne-universite.fr (E.J.); renaud.delaunay@sorbonne-universite.fr (R.D.); romain.jarrier@sorbonne-universite.fr (R.J.); gheorghe.chiuzbaian@sorbonne-universite.fr (G.S.C.); jan.luning@helmholtz-berlin.de (J.L.)

2 Institut Jean Lamour (UMR 7198), Université de Lorraine, Centre National de la Recherche Scientifique (CNRS), 54011 Nancy, France; gregory.malinowski@univ-lorraine.fr

3 Deutsches Elektronen-Synchrotron, 22607 Hamburg, Germany; torsten.golz@class5photonics.com (T.G.); ekaterina.zapolnova@class5photonics.com (E.Z.); rui.pan@desy.de (R.P.); nikola.stojanovic@dlr.de (N.S.)

4 DLR-Institute for Optical Sensor Systems, 12489 Berlin, Germany

* Correspondence: boris.vodungbo@sorbonne-universite.fr

† Current address: Helmholtz-Zentrum Berlin für Materialien und Energie, Hahn-Meitner-Platz 1, 14109 Berlin, Germany.

check for updates

Citation: Liu, X.; Jal, E.; Delaunay, R.; Jarrier, R.; Chiuzbaian, G.S.;

Malinowski, G.; Golz, T.; Zapolnova,

E.; Pan, R.; Stojanovic, N.; et al.

Investigating Coherent Magnetization Control with Ultrashort THz Pulses.

Appl. Sci. 2022, 12, 1323. https://

doi.org/10.3390/app12031323

Academic Editor: Emiliano Principi

Received: 21 December 2021

Accepted: 19 January 2022

Published: 26 January 2022

Publisher's Note: MDPI stays neutral with regard to jurisdictional claims in published maps and institutional affiliations.

Copyright: (c) 2022 by the authors. Licensee MDPI, Basel, Switzerland. This article is an open access article distributed under the terms and conditions of the Creative Commons Attribution (CC BY) license (https:/ / creativecommons.org/licenses/by/ $4.0 /)$.

\begin{abstract}
Coherent terahertz control of magnetization dynamics is an area of current interest due to its great potential for the realization of magnetization control on ultrafast timescales in commercial devices. Here we report on an experiment realized at the $\mathrm{THz}$ beamline of the free electron laser FLASH at DESY which offers a tunable terahertz radiation source and spontaneously synchronized free-electron laser X-ray pulses to resonantly probe the magnetization state of a ferromagnetic film. In this proof-of-principle experiment, we have excited a thin Permalloy film at different $\mathrm{THz}$ wavelengths and recorded the induced magnetization dynamics with photons resonantly tuned to the $\mathrm{Ni} M_{2,3}$ absorption edge. For THz pump pulses including higher orders of the undulator source we observed demagnetization dynamics, which precise shape depended on the employed fundamental wavelength of the undulator source. Analyzing the shape in detail, we can reconstruct the temporal profile of the electric field of the THz pump pulse. This offers a new method for the realization of an in-situ terahertz beamline diagnostic which will help researchers to adjust the pulse characteristics as needed, for example, for future studies of $\mathrm{THz}$ induced coherent control of magnetization dynamics.
\end{abstract}

Keywords: X-ray free electrons laser; ultrafast demagnetization; terahertz radiations

\section{Introduction}

One of the main drives for ultrafast magnetization dynamics studies is applications in magnetic storage technology and logic operations with spintronic devices [1]. For example, breaking the current nanosecond time scale limit for magnetization manipulation would allow to create ultrafast magnetic devices as needed for future Big Data or Artificial Intelligence applications. To achieve this goal, it is not sufficient to only demagnetize a system as demonstrated on the femtosecond time scale in 1996 [2]; instead, it is necessary to completely control its magnetization. In that regard, the experiment realized by Vicario et al. [3] is remarkable. Using a terahertz (THz) pump, these authors were able to initiate a non resonant precession of the magnetization in a thin cobalt film on the time scale of a picosecond. This observation opens up the way to the deterministic control of magnetization with ultrashort pulses.

$\mathrm{THz}$ pulses have a longer wavelength than infrared (IR) pulses, hence a lower frequency. Consequently, the THz photon energy is lower (by almost three orders of mag- 
nitude) than that of a near IR photon which is generally used as pump in ultrafast magnetization experiments $(0.004 \mathrm{eV}$ versus $1.6 \mathrm{eV})$. Therefore, absorption of a $\mathrm{THz}$ photon can only occur by electrons very close to the Fermi level and only into lower energy levels than it is the case of an IR pump pulse (Figure 1). The THz pulse induced dynamics is then dominated by the interaction between the sample and the strong magnetic field of the $\mathrm{THz}$ pulse rather than by absorption and heating. An interesting question arises therefore, at which wavelength, or over which wavelengths range, we can observe the transition between the two behaviors. This knowledge will be fundamental to tailor ultrafast magnetic devices. Our goal is thus to study the transition from such a coherent coupling to the non-coherently excited ultrafast demagnetization phenomenon.

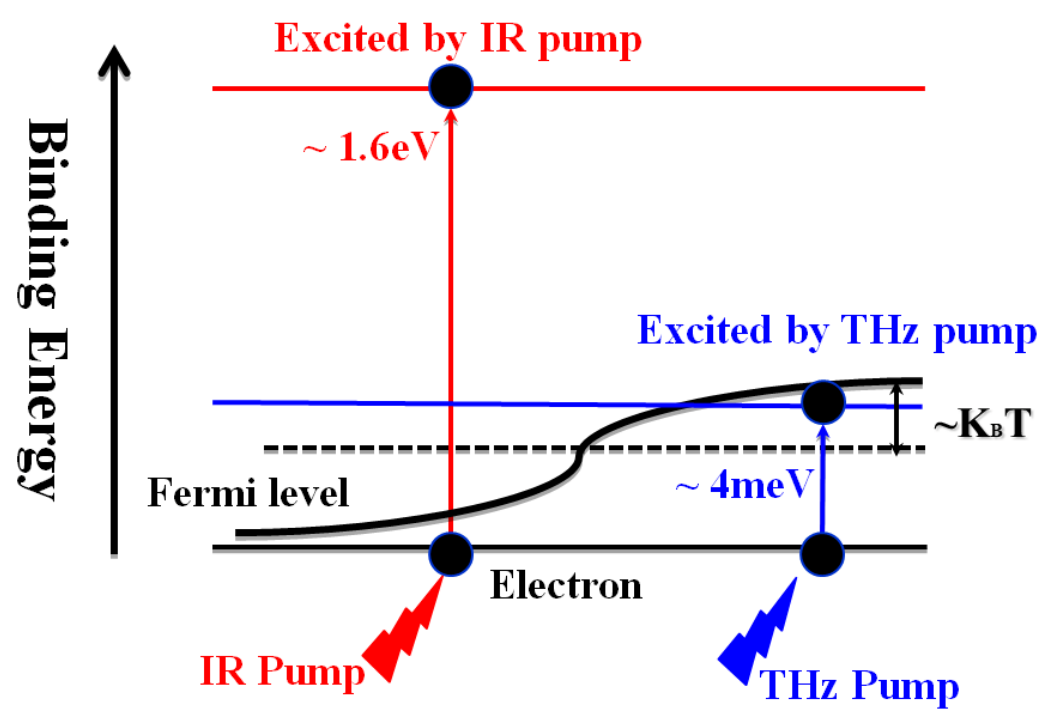

Figure 1. Comparison between IR pump and THz pump in terms of photon energy excitation.

Recently, the generation of $\mathrm{THz}$ pulses with much higher field amplitudes has become possible, which permitted a conceptually new approach for investigating low energy degrees of freedom in matter [4]. Additionally, advancements of accelerator technology achieved, for example at BL3 beamline of FLASH (DESY, Hamburg, Germany), made it possible to produce carrier-envelope phase stable $\mathrm{THz}$ pulses with high fields and adjustable high repetition rates [5]. Appropriate parameters settings of the $\mathrm{THz}$ undulator allows for the generation of narrow-band $\mathrm{THz}$ frequency and intense pulses, which can be used to trigger magnetization dynamics coherently.

In the present work, we use synchronized THz-pump to an X-ray Free Electrons Laser (XFEL) source to investigate the THz induced magnetization dynamics in a Permalloy (Py) film. The tunable $\mathrm{THz}$ undulator allows us to select the pulse duration and the wavelength. A filter is used to remove higher orders generated by the undulator and to obtain a single frequency $\mathrm{THz}$ pulse. The extreme ultraviolet (XUV) radiation delivered by the XFEL, a powerful tool for probing magnetic phenomena at the nanometer and femtosecond scales [6-9], is used to study the resonant magneto-optic response at the $\mathrm{Ni} M_{2,3}$ absorption edges (around $66.7 \mathrm{eV}$ ) [10]. Unfortunately, we did not succeed to achieve any ultrafast coherent magnetization control by the THz pump with a fundamental frequency of $100 \mu \mathrm{m}$ because the $\mathrm{THz}$ intensity was too low.

However, after the removal of the filter from the $\mathrm{THz}$ undulator beam path, an ultrafast demagnetization of the Py film can be evidenced. The pump radiation is now composed of the fundamental frequency but also higher harmonics, that span from $\mathrm{THz}$ to IR wavelength range, which are responsible for this non coherent dynamics. The dynamics is more complex than the usual demagnetization response and allowed us to reconstruct the shape of the fundamental $\mathrm{THz}$ electric field. This method will provide an in-situ and fast way to obtain a characterization of the THz pulse, which is fundamental for the 
interpretation of future coherent magnetization control experiments, in addition to more conventional characterization methods which can be difficult to implement during an experiment $[5,11]$.

\section{Materials and Methods}

$\mathrm{THz}$ radiation is produced by the $\mathrm{THz}$ undulator of the BL3 beamline at FLASH, a purpose built planar electromagnetic undulator with 9 full periods of $40 \mathrm{~cm}$ [12]. It can generate radiation in a very broad spectral range from the mid infrared to the far infrared spectral regions corresponding to wavelengths between $1 \mu \mathrm{m}$ and $300 \mu \mathrm{m}$ (respectively $300 \mathrm{THz}$ and $1 \mathrm{THz}$ ). The pulse duration and intensity depends on the wavelength chosen: the $\mathrm{THz}$ pulse has about ten optical cycles (corresponding to a pulse duration of about $33.3 \mathrm{fs}$ and $10 \mathrm{ps}$ respectively) and the maximum pulse energy is $150 \mu \mathrm{J}$ [5].

The $\mathrm{THz}$ undulator is implemented in series, and can be operated simultaneously to the XUV undulator of FLASH for the realization of THz pump-XUV probe experiments (see Figure 2). To do so, an electron bunch is accelerated in the tunnel, passes into the XUV undulator and generates an XUV pulse. The electrons bunch then passes through the $\mathrm{THz}$ undulator and generates a $\mathrm{THz}$ pulse. Those two pulses are separated by a holey mirror and then recombine onto the sample thanks to a series of mirrors. Since both THz and XUV pulses are emitted from the same electron bunch, they are inherently synchronized [5,12].

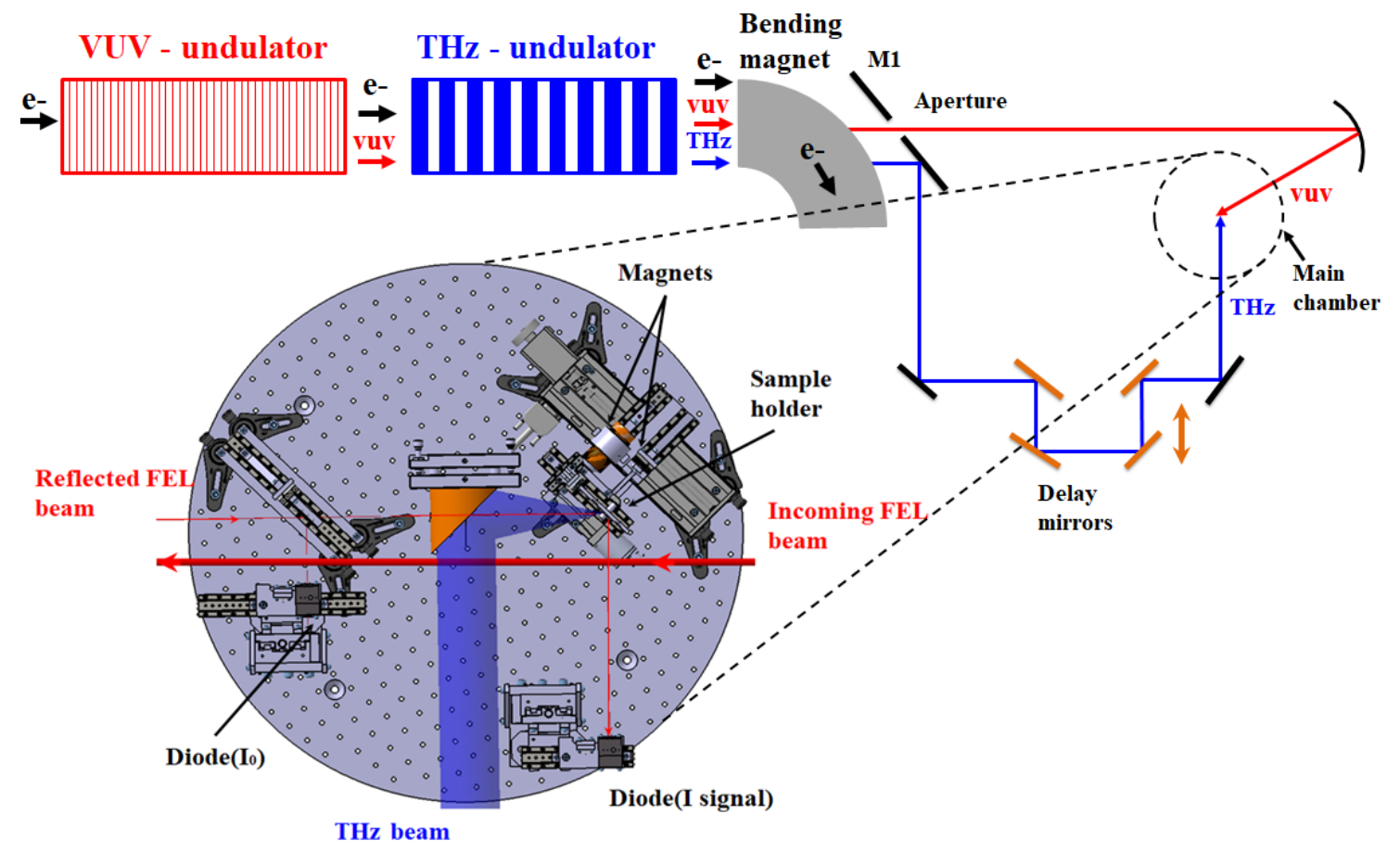

Figure 2. Layout of the experimental setup. XUV probe pulse (red) and THz pump pulse (blue) are produced by the XUV undulator and the THz undulator respectively. The zoom on the main chamber shows the $\mathrm{THz}$ beam focused onto the sample by a parabola and the probe beam going through the chamber before coming back focused onto the sample by a spherical mirror (not shown). Two APDs are used to measure the intensity of the incoming probe beam (measuring the partial reflection on a $\mathrm{Si}_{3} \mathrm{~N}_{4}$ window) and the signal reflected by the sample.

In more detail, the experimental setup consists of two vacuum chambers. The incoming XUV beam (red in Figure 2) passes through the first chamber and is reflected back and focused by a spherical mirror in the second chamber (not shown in the Figure 2). The tilts of this back mirror are motorized in order to be able to change the position of the beam on the sample. This allow us to find the spatial overlap of the probe beam with the $\mathrm{THz}$ beam. The reflected and focused XUV beam comes back to the main chamber, passes 
through a center aperture in the THz off-axis parabola (orange in the zoom of Figure 2) and eventually arrives on the sample. In the meantime, the $\mathrm{THz}$ beam enters the main chamber perpendicularly to the XUV beam and is focused onto the sample by the off-axis parabola. The exact spots sizes on the sample could not be measure in-situ but the characteristics of the beams and the focusing mirrors should yield a focal spot diameter of lower than $100 \mu \mathrm{m}$ for XUV and larger than $400 \mu \mathrm{m}$ for THz ensuring a uniform excitation of the probed area.

To probe the magnetization dynamics, we used the time-resolved Transverse MagnetoOptical Kerr Effect (T-MOKE). The basic principles of this technique have been presented in many reports [13-15]. The geometry is represented in Figure 3. The incident angle on the sample is chosen to be close to $45^{\circ}$ to maximize the T-MOKE signal. Two avalanche photodiodes (APD) measure the XUV beam incoming intensity, $I_{0}$, and the reflected signal, $I_{\mathrm{r}}$, from the sample. An electromagnet is used to magnetize the sample in plane in two opposite directions, $M_{+}$and $M_{-}$, giving rise to two different signal intensities, $I_{+}$and $I_{-}$, measured as $\frac{I_{\mathrm{r}}}{I_{0}}$ for positive and negative magnetizations respectively.

The sample studied consisted of a $10 \mathrm{~nm}$ thin Py film sputtered on a silicon substrate with a $3 \mathrm{~nm}$ thin Ta buffer and capped with a $3 \mathrm{~nm}$ thin Al layer to prevent oxidation. To measure the magnetization dynamics of this sample, we tuned the XFEL to a photon energy of $66.6 \mathrm{eV}$, in resonance with the $M_{2,3}$ absorption edges of $\mathrm{Ni}$, and recorded $I_{+}$and $I_{-}$for delays between the THz pump and the XUV probe ranging from $-5 \mathrm{ps}$ to $15 \mathrm{ps}$.

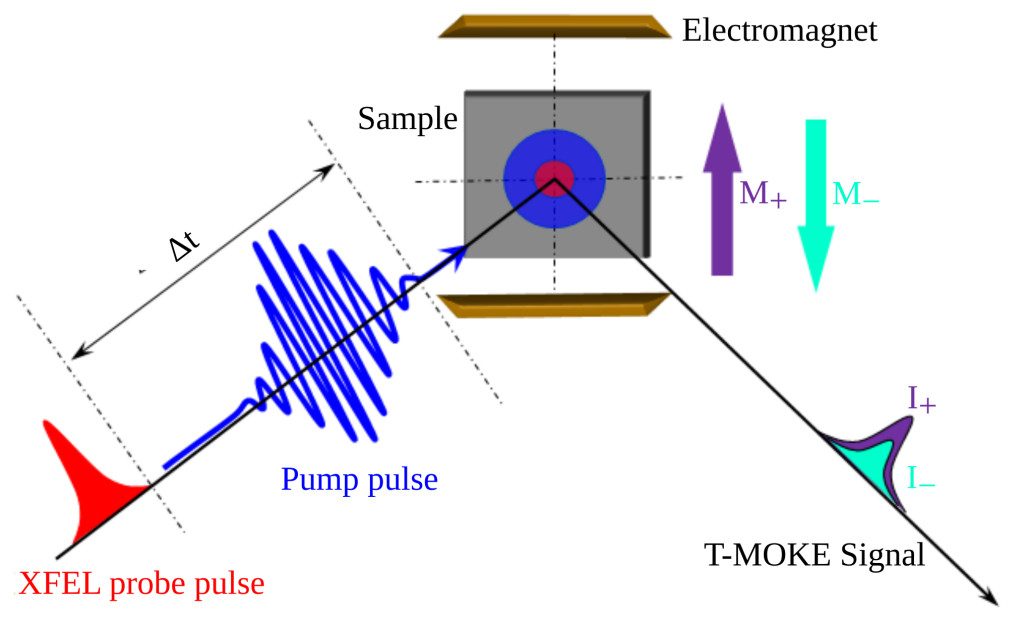

Figure 3. Time resolved T-MOKE configuration showing the probe (red) and pump (blue) beams, their footprints on the sample (red and blue spots) which is situated in the air gap of an electromagnet. The two opposite directions of magnetization $\left(M_{+}\right.$and $\left.M_{-}\right)$of the sample are represented (violet and cyan arrows) as well as the corresponding pulse intensities $I_{+}$and $I_{-}$(violet and cyan envelopes).

\section{Results}

First, we tuned the $\mathrm{THz}$ undulator to obtain a fundamental wavelength of $100 \mu \mathrm{m}$. To suppress higher harmonics, we used a $50 \mu \mathrm{m}$ long-pass filter. This should results in a pump pulse consisting of about 10 oscillations of a $3 \mathrm{THz}$ electromagnetic field and a pulse duration of about 3.3 ps. The coupling between the magnetic field of this radiation and the magnetization of the sample should results in oscillations in the magnetic signal with a period of about $333 \mathrm{fs}$ (the period of the $\mathrm{THz}$ radiation and hence of the associated magnetic field). From previous experiments [3], a THz fluence of about $10 \mathrm{~mJ} \mathrm{~cm}^{-1}$ should be sufficient to trigger this effect $\left(1 \mathrm{~mJ} \mathrm{~cm}^{-1}\right.$ were enough for a single cycle $2.1 \mathrm{THz}$ pulse). 
Figure 4a shows the sample reflectivity as function of time delay for the two opposite magnetization direction of the sample. Because of T-MOKE, the reflectivity for $M_{+}$is almost 3 times higher than the reflectivity for $M_{-}$. However, there is no effect of the $\mathrm{THz}$ field on the magnetization of the sample. After investigating at different $\mathrm{THz}$ wavelengths we came to the conclusion that the magnetic field associated to the THz pump was too weak to induce a magnetic response. This was most probably due to a combination of two factors: the intensity in the fundamental was a little lower than expected and the focal spot was too large to compensate and obtain the right fluence.

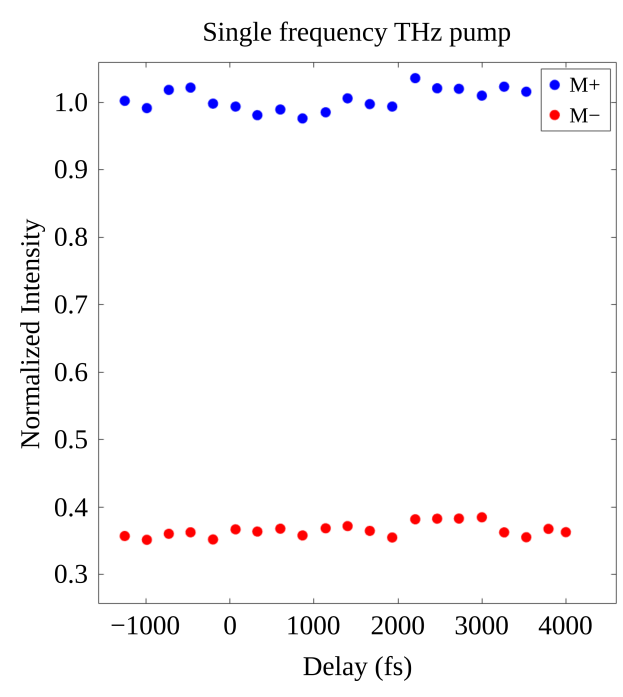

(a)

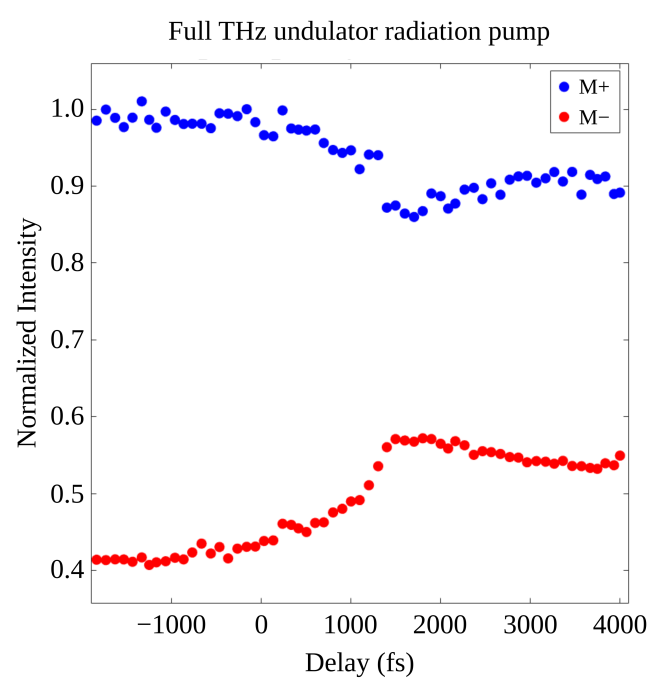

(b)

Figure 4. Normalized T-MOKE intensity for two opposite magnetization directions (a) for a single frequency $\mathrm{THz}$ pump ( $3 \mathrm{THz}$ ) and (b) for a pump consisting of the complete radiation content delivered by the $\mathrm{THz}$ undulator (fundamental frequency of $6.7 \mathrm{THz}$ ). In the first case, the magnetization does not change while in the latter case the pump triggers a modification of the magnetization on a timescale of a few ps.

To increase the pump pulse intensity, we removed the long-pass filter. This should increase the pulse intensity by a factor of at least 4 [5]. By doing so, we were able to trigger a modification of the sample magnetization state with the THz pump (Figure $4 \mathrm{~b}$ ). To analyze this magnetization dynamics in more detail, we have plotted the so-called asymmetry, defined as $A=\frac{I_{+}-I_{-}}{I_{+}+I_{-}}$, in Figure 5 for two different THz fundamental wavelength, 45 and $100 \mu \mathrm{m}$. The curves appear very similar to the typical curves obtained by performing a demagnetization experiment with femtosecond IR pulses.

The magnetization dynamics is nonetheless very different to the typical ultrafast demagnetization in transition metals thin films $[16,17]$. First, as seen on Figure 5, the demagnetization occurs on a much longer timescale of a few picoseconds. Moreover, the timescale of the dynamics depends on the fundamental wavelength of the $\mathrm{THz}$ pump. Finally, the demagnetization curves appear to be the results of several different demagnetization processes triggered successively. All together, these point out to the fact that our measurements are dominated by the shape of the $\mathrm{THz}$ pulse. 


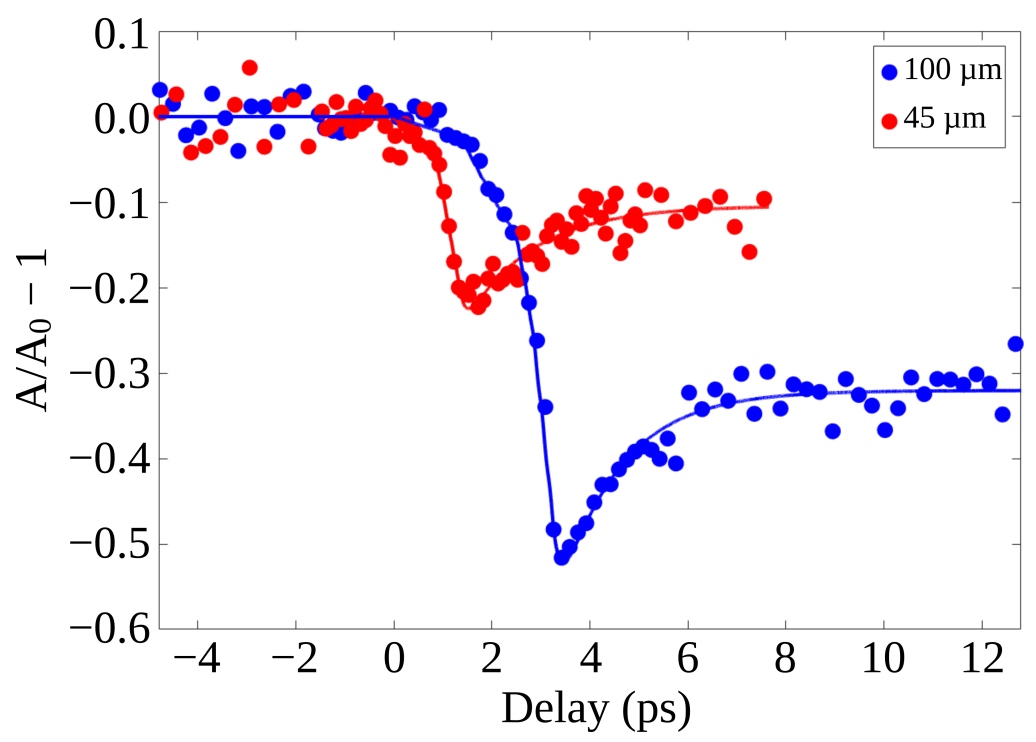

Figure 5. Normalized asymmetry as a function of time delay for $\mathrm{THz}$ pump of fundamental wavelengths of $45 \mu \mathrm{m}$ (red) and $100 \mu \mathrm{m}$ (blue) as a function of time delay. The unusually long demagnetization time is a consequence of the $\mathrm{THz}$ pulse length of about 1.5 and 3.3 ps respectively. The exact shape of the pump pulse gives rise to the varying slope in the demagnetization trace.

To characterize this pulse shape, we fitted our data with the following equation which is often used to describe ultrafast demagnetization $[7,18]$ :

$$
\frac{A-A_{0}}{A_{0}}=\left[\left(K_{1}-\frac{\left(K_{2} \tau_{E}-K_{1} \tau_{M}\right) e^{\frac{-t}{\tau_{M}}}}{\tau_{E}-\tau_{M}}-\frac{\tau_{E}\left(K_{1}-K_{2}\right) e^{\frac{-t}{\tau_{E}}}}{\tau_{E}-\tau_{M}}\right) H(t)\right] * \Gamma(t),
$$

where $A_{0}$ is the unpumped asymmetry, $K_{1}$ and $K_{2}$ are constants, $\tau_{E}$ and $\tau_{M}$ are the electronsphonons and spins relaxation times, $H(t)$ the Heaviside function and $\Gamma(t)$ the temporal intensity profile of the pump beam. To simplify the analysis, we considered that the pump beam consisted of 10 cycles (given the number of period of the $\mathrm{THz}$ undulator) of a single $\mathrm{THz}$ frequency of either 3 or $6.7 \mathrm{THz}$ (corresponding to pulse durations of about $3.3 \mathrm{ps}$ and 1.5 ps respectively). This ignores the fact that the higher harmonics will distort the shape of the $\mathrm{THz}$ pulse [11] but a more accurate description would not match the time resolution of our data.

The results of the fits are shown in Figure 6. The most evident observation is that the intensity is not uniformly spread among cycles for both $\mathrm{THz}$ pump wavelengths. This was to be expected by observing the demagnetization curves on Figure 5 which presented broken lines demagnetization slopes. Moreover, the most intense cycles are not situated at the same position within the pulse for 45 and $100 \mu \mathrm{m}$ fundamental wavelengths: respectively in the middle and at the end of the $\mathrm{THz}$ pulse. This observations are in rough agreement with proper measurements of the $\mathrm{THz}$ pulse shape which showed that the exact shape of the THz pulse is highly dependent on the XFEL parameters and can drastically change upon variation of these parameters during an experiment [5]. 


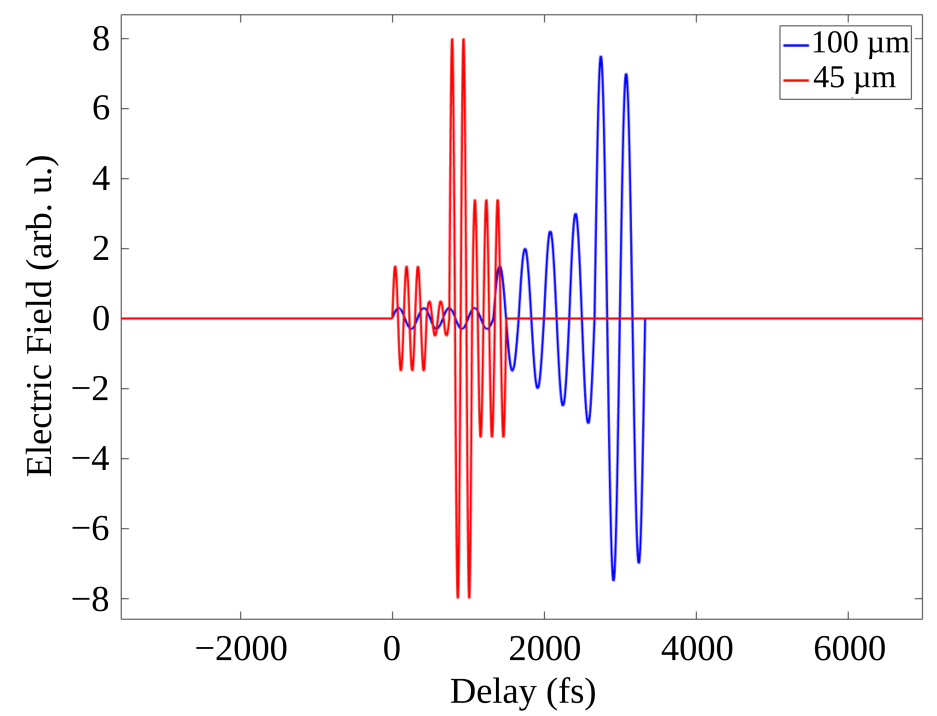

Figure 6. Reconstructed electric field of the $\mathrm{THz}$ radiation obtained by fitting the results of Figure 5 by Equation (1) for fundamental wavelengths of $45 \mu \mathrm{m}$ (red) and $100 \mu \mathrm{m}$ (blue). The fit neglects the higher harmonics for simplicity. The intensity is not uniformly spread among cycles and the most intense cycles are not located at the same position within the pulse for the two different wavelengths.

\section{Discussion}

Given the pump fluence of our experiment we were not able to trigger a detectable coherent magnetization dynamics with a single frequency $\mathrm{THz}$ radiation pump pulse. Adding higher harmonics to the pump pulse resulted in a clearly visible reduction of the sample's magnetization occurring on the picosecond timescale. However, there is no signature of the magnetization to be directly affected by the magnetic field of the THz pump pulse, the observed dynamics appears to be driven by a pure demagnetization process. This observation indicates that the magnetic field strength has been too low at any given time to trigger a coherent response of the sample's magnetization, while incoherent energy transfer through energy absorption accumulating over the successive cycles of the $\mathrm{THz}$ pump pulse induced the observed demagnetization dynamics. To be able to observe the $\mathrm{THz}$ induced coherent magnetization dynamics in a follow-up experiment, it is therefore necessary to increase the $\mathrm{THz}$ fluence, which can be achieved by improving the focusing of the $\mathrm{THz}$ beam.

Our results also showed that the THz pulse envelope emitted by the FLASH undulator source is highly non-uniformly structured. This indicates that an analysis of an experiment of coherent $\mathrm{THz}$ control of magnetization dynamics at this source will require a detailed characterization of the $\mathrm{THz}$ pulse shape. Such a diagnostic is not yet permanently accessible at the $\mathrm{THz}$ beamline of FLASH, but the results reported here provide due to the rather simple setup an attractive way to do so.

Author Contributions: J.L. conceived the experiment with help from R.J., N.S. and B.V., R.J., J.L. and N.S. designed the experimental set-up. R.D. prepared the samples. T.G., E.Z., R.P. and N.S. operated the beamline. X.L., G.S.C., G.M., T.G., E.Z., R.P., N.S., J.L. and B.V. conducted the experiment. X.L. and B.V. analyzed the data. X.L. and B.V. prepared the original draft. E.J., N.S., J.L. and B.V. reviewed and edited the text. All authors have read and agreed to the published version of the manuscript.

Funding: This research was funded by the CNRS PICS (now IEA) program (STEROID), the Sorbonne Université émergence program (MADAM), the ANR under grant ANR-15-CE24-0009 and the CNRS MOMENTUM program.

Institutional Review Board Statement: Not applicable.

Informed Consent Statement: Not applicable. 
Data Availability Statement: The data presented in this study are available on request from the corresponding author.

Acknowledgments: We acknowledge fruitful discussions with Valentin Chardonnet, Marcel Hennes, Jérémie Caillat, Jérôme Palaudoux, Loïc Journel and David Massot.

Conflicts of Interest: The authors declare no conflict of interest.

\section{References}

1. Bader, S.D.; Parkin, S.S.P. Spintronics. Annu. Rev. Condens. Matter Phys. 2010, 1, 71-88. [CrossRef]

2. Beaurepaire, E.; Merle, J.C.; Daunois, A.; Bigot, J.Y. Ultrafast spin dynamics in ferromagnetic nickel. Phys. Rev. Lett. 1996, 76, 4250. [CrossRef] [PubMed]

3. Vicario, C.; Ruchert, C.; Ardana-Lamas, F.; Derlet, P.M.; Tudu, B.; Luning, J.; Hauri, C.P. Off-resonant magnetization dynamics phase-locked to an intense phase-stable terahertz transient. Nat. Photonics 2013, 7, 720-723. [CrossRef]

4. Kampfrath, T.; Battiato, M.; Maldonado, P.; Eilers, G.; Nötzold, J.; Mährlein, S.; Zbarsky, V.; Freimuth, F.; Mokrousov, Y.; Blügel, S.; et al. Terahertz spin current pulses controlled by magnetic heterostructures. Nat. Nanotechnol. 2013, 8, 256-260. [CrossRef] [PubMed]

5. Pan, R.; Zapolnova, E.; Golz, T.; Krmpot, A.J.; Rabasovic, M.D.; Petrovic, J.; Asgekar, V.; Faatz, B.; Tavella, F.; Perucchi, A.; et al. Photon diagnostics at the FLASH THz beamline. J. Synchrotron Radiat. 2019, 26, 700-707. [CrossRef] [PubMed]

6. Graves, C.E.; Reid, A.H.; Wang, T.; Wu, B.; de Jong, S.; Vahaplar, K.; Radu, I.; Bernstein, D.P.; Messerschmidt, M.; Müller, L.; et al. Nanoscale spin reversal by non-local angular momentum transfer following ultrafast laser excitation in ferrimagnetic GdFeCo. Nat. Mater. 2013, 12, 293-298. [CrossRef] [PubMed]

7. Hennes, M.; Merhe, A.; Liu, X.; Weder, D.; Schmising, C.v.K.; Schneider, M.; Günther, C.M.; Mahieu, B.; Malinowski, G.; Hehn, M.; et al. Laser-induced ultrafast demagnetization and perpendicular magnetic anisotropy reduction in a $\mathrm{Co}_{88} \mathrm{~Tb}_{12}$ thin film with stripe domains. Phys. Rev. B 2020, 102, 174437. [CrossRef]

8. Jal, E.; Makita, M.; Rösner, B.; David, C.; Nolting, F.; Raabe, J.; Savchenko, T.; Kleibert, A.; Capotondi, F.; Pedersoli, E.; et al. Single-shot time-resolved magnetic X-ray absorption at a free-electron laser. Phys. Rev. B 2019, 99, 144305. [CrossRef]

9. Pfau, B.; Schaffert, S.; Müller, L.; Gutt, C.; Al-Shemmary, A.; Büttner, F.; Delaunay, R.; Düsterer, S.; Flewett, S.; Frömter, R.; et al. Ultrafast optical demagnetization manipulates nanoscale spin structure in domain walls. Nat. Commun. 2012, 3, 1-6. [CrossRef] [PubMed]

10. Hofherr, M.; Häuser, S.; Dewhurst, J.K.; Tengdin, P.; Sakshath, S.; Nembach, H.T.; Weber, S.T.; Shaw, J.M.; Silva, T.J.; Kapteyn, H.C.; et al. Ultrafast optically induced spin transfer in ferromagnetic alloys. Sci. Adv. 2020, 6, eaay8717. [CrossRef] [PubMed]

11. Golz, T. High Repetition Rate THz Characterization at 4th Generation X-ray Light Sources. Ph.D. Thesis, University Hamburg, Hamburg, Germany, 2019. Available online: https://inis.iaea.org/search/search.aspx?orig_q=RN:50010978 (accessed on 18 January 2022)

12. Gensch, M.; Bittner, L.; Chesnov, A.; Delsim-Hashemi, H.; Drescher, M.; Faatz, B.; Feldhaus, J.; Fruehling, U.; Geloni, G.A.; Gerth, C.; et al. New infrared undulator beamline at FLASH. Infrared Phys. Technol. 2008, 51, 423-425. [CrossRef]

13. Vodungbo, B.; Gautier, J.; Lambert, G.; Zeitoun, P.; Lüning, J. Comment on “Ultrafast Demagnetization Measurements Using Extreme Ultraviolet Light: Comparison of Electronic and Magnetic Contributions". Phys. Rev. X 2013, 3, 038001. [CrossRef]

14. Buzzi, M.; Makita, M.; Howald, L.; Kleibert, A.; Vodungbo, B.; Maldonado, P.; Raabe, J.; Jaouen, N.; Redlin, H.; Tiedtke, K.; et al. Single-shot Monitoring of Ultrafast Processes via X-ray Streaking at a Free Electron Laser. Sci. Rep. 2017, 7, 7253. [CrossRef] [PubMed]

15. La-O-Vorakiat, C.; Siemens, M.; Murnane, M.M.; Kapteyn, H.C.; Mathias, S.; Aeschlimann, M.; Grychtol, P.; Adam, R.; Schneider, C.M.; Shaw, J.M.; et al. Ultrafast Demagnetization Dynamics at the M Edges of Magnetic Elements Observed Using a Tabletop High-Harmonic Soft X-Ray Source. Phys. Rev. Lett. 2009, 103, 257402. [CrossRef] [PubMed]

16. Alves, C.; Lambert, G.; Malka, V.; Hehn, M.; Malinowski, G.; Hennes, M.; Chardonnet, V.; Jal, E.; Lüning, J.; Vodungbo, B. Resonant Faraday effect using high-order harmonics for the investigation of ultrafast demagnetization. Phys. Rev. B 2019, 100, 144421. [CrossRef]

17. Koopmans, B.; Malinowski, G.; Dalla Longa, F.; Steiauf, D.; Fähnle, M.; Roth, T.; Cinchetti, M.; Aeschlimann, M. Explaining the paradoxical diversity of ultrafast laser-induced demagnetization. Nat. Mater. 2010, 9, 259-265. [CrossRef] [PubMed]

18. Malinowski, G.; Dalla Longa, F.; Rietjens, J.H.H.; Paluskar, P.V.; Huijink, R.; Swagten, H.J.M.; Koopmans, B. Control of speed and efficiency of ultrafast demagnetization by direct transfer of spin angular momentum. Nat. Phys. 2008, 4, 855-858. [CrossRef] 